\title{
Prevención de trastorno de estrés postraumático en supervivientes del terremoto de Chile de febrero de 2010: una propuesta de intervención narrativa
}

\section{Prevention of disorder of posttraumatic stress in survivors of the earthquake of Chile of february, 2010: an offer of narrative intervention}

\author{
Felipe E. García \\ Universidad de las Américas y Universidad Pedro de Valdivia, Chile \\ Rodrigo Mardones \\ Universidad de Las Américas, Chile
}

(Rec: 01 abril 2010 / Acep: 14 mayo 2010)

\begin{abstract}
Resumen
Tras el terremoto y tsunami que afectaron a la zona centro sur de Chile el 27 de febrero de 2010, se hace necesario anticipar las consecuencias psicológicas que este tipo de desastres provoca en la población. El presente trabajo revisa los resultados de distintos estudios a nivel mundial a fin de conocer estas consecuencias, centrándose principalmente en el Trastorno de Estrés Postraumático [TEPT], sus características particulares, prevalencia, factores de riesgo e intervenciones utilizadas para su prevención y tratamiento. A continuación, se propone un modelo de intervención narrativa, enriquecido por los aportes de los estudios sobre expresión emocional y apoyo social en la prevención de este trastorno. Esta propuesta se ha probado anteriormente con éxito para la prevención del TEPT y sintomatología asociada en mujeres con cáncer de mama.

Palabras clave: Terremoto - Terapia narrativa - Estrés postraumático.
\end{abstract}

\begin{abstract}
After the earthquake and tsunami that struck the south-central Chile on February 27, 2010, it is necessary to anticipate the psychological consequences of disasters such causes in the population. This paper reviews the results of various studies worldwide in order to understand these consequences, focusing mainly on posttraumatic stress disorder [PTSD], their characteristics, prevalence, risk factors and interventions used for prevention and treatment. Then, we propose a model of narrative intervention, enriched by the contributions of studies on emotional expression and social support in the prevention of this disorder. This proposal has been tried before successfully for the prevention of PTSD and associated symptoms in women with breast cancer.
\end{abstract}

Key words: Earthquake - Narrative therapy - Post-traumatic stress disorder. 


\section{Introducción}

A las 03:34 de la madrugada del día 27 de febrero de 2010, la región centro sur de Chile fue devastada por un terremoto magnitud 8,8 en la escala de Richter. Tras el sismo, varios tsunamis arrasaron la costa. Hasta el momento en que se redacta este artículo se contabilizan 486 muertos y 79 desaparecidos. La catástrofe no fue solo natural, la desorganización central y el colapso de las comunicaciones suscitaron un caos que llevó a saqueos de propiedad pública y privada. La indefensión se intensificó ante la falta de ayuda oportuna, el retraso en la reposición de servicios básicos, constantes réplicas que han alcanzado hasta los 7 grados de magnitud y la influencia de los medios de comunicación, que informan hasta hoy de un probable nuevo gran terremoto.

El carácter traumático de un evento de esta naturaleza es innegable. Sin embargo, las respuestas psicológicas de los supervivientes tienden a ser más variadas de lo que la gente supone. En tal sentido, es necesario resaltar que, tal como afirma Viktor Frankl en su libro "El hombre en busca de sentido": ante una situación anormal, la reacción anormal constituye una conducta normal. La necesidad de normalizar las reacciones psicológicas en un primer contacto psicoterapéutico con los supervivientes pasa a ser entones un paso importante para ayudar en el proceso de adaptación.

Según Gaborit (2001), a diferencia de otros desastres naturales, los terremotos tienen como característica la dificultad de su predicción. Siempre toma por sorpresa, su duración es breve, de gran intensidad y de un poder destructivo inigualable, generando un fuerte sentimiento de indefensión, ansiedad e incertidumbre cognitiva. $A s u$ vez, las constantes réplicas crean las condiciones para la reexperimentación del trauma inicial.

Asimismo, Gaborit (2006) resalta que frente a un terremoto, las rutinas individuales se ven súbitamente alteradas por tiempo impredecible. Muchos planes y proyectos, muchos estilos de vida han debido cambiar como consecuencia del sismo. La necesidad de adaptarse es fuerte y al parecer no todos están preparados para hacerlo.

Eventos como los desastres naturales fijan un punto de referencia en las vidas de quienes lo sufren, la catástrofe parte el propio discurso biográfico en un antes y un después, constituyendo un verdadero quiebre narrativo. Sassón (2004) advierte que en los desastres se rompen creencias básicas acerca de la propia invulnerabilidad, la creencia de que la vida tiene un sentido y la creencia de que los sucesos son todos ordenados, predecibles y controlables. Janoff-Bulman (1989, 1992, citado en Páez, Arroyo \& Fernández, 1995) señala que en general los individuos poseen una visión global de nuestra realidad basada en cuatro creencias: ilusión de control del mundo, creencia en que el mundo es justo, creencia en que los sucesos se reparten por azar y creencia en que los demás y el mundo es bueno.
Esta cosmogonía se ve muy afectada ante una catástrofe lo que no sólo genera consecuencias psicológicas, sino que además, cambia la visión de sí mismos, del mundo y de los otros, hacia una mirada más negativa. Janoff-Bulman apunta a que las víctimas de catástrofes creen menos en que el mundo tiene sentido y pierden la ilusión de control. Sin embargo, concluye en que con el paso del tiempo las personas terminan por readaptarse. En las Regiones de Bío Bío y Maule, es posible afirmar que hubo dos tipos de catástrofe: uno de índole natural, el terremoto y posterior tsunami, y una catástrofe humana, provocada por los saqueos. Ambas con gran poder destructivo y traumático. Y ambas golpearon la estructura de creencias de la población.

La literatura científica logra cierto consenso al momento de identificar fases en la reacción de la población después de una catástrofe natural. Páez et al., (1995) sintetizan estas fases del siguiente modo: a) una primera fase caracterizada por la sensación de víctima y abandono que sufren los afectados; b) una segunda fase, caracterizada por conductas adaptativas tales como toma de distancia del hecho, búsqueda de una explicación para entender lo ocurrido, expresión emocional y las primeras acciones destinadas a protegerse del peligro, aunque también aparecen el pánico colectivo, la huida y la queja; c) una tercera fase en la que se incrementan los rumores, se recupera la autoestima, se ayuda a los demás y se restituye el control perdido de la situación; d) finalmente, en la cuarta fase, o período postcrítico, dependiendo de los sujetos en particular y del apoyo social del que dispongan, de sus creencias acerca del mundo y de las formas de afrontamiento, pueden manifestar dos tendencias opuestas, unos piensan el evento y lo rumian y otros desarrollan conductas evitativas, rechazando el recuerdo y hablar de lo ocurrido.

Desde otra perspectiva, Marcos, Medina, Pérez-Iñigo, Robles y Gómez-Trigo (2002) clasifican las reacciones posibles ante una catástrofe en tres tipos: a) reacciones adaptadas, caracterizadas por la capacidad de mantener la calma, se toman medidas de cuidado y protección, se observan a veces conductas de solidaridad y auxilio a otros; $b$ ) reacciones inadaptadas, que corresponden a las conductas de pánico, desborde emocional, inhibición, estupor, negación y oposición; c) reacciones influenciables, personas que se muestran inseguras e indecisas y que actúan según las circunstancias, por lo tanto, pueden ser movilizados a la ayuda o hacia un sentido más negativo como la inhibición, pánico $\mathrm{y}$ fuga. Son las personas a las que se dirigen los esfuerzos informativos y de entrenamiento.

En cuanto a la posibilidad de sufrir un Trastorno de Estrés Postraumático [TEPT] a consecuencia de un terremoto, Shinfuko (2002) sugiere que éste se debe considerar como parte de la variedad de problemas de salud mental entre las víctimas. Ansiedad, problemas para dormir, depresión, conductas autodestructivas como el abuso de alcohol, son también comunes entre las víctimas. Shinfuku (2002) 
agrega que TEPT fue la palabra más utilizada para referirse a los problemas mentales tras el terremoto de Kobe, Japón, en 1995, el concepto fue ampliamente utilizado y aceptado por los medios de comunicación japoneses y se utilizó como sinónimo de toda la gama de problemas psicológicos.

Quizás esta confusión, sumada a problemas relativos a los instrumentos y al acceso a la población afectada, puede explicar la variabilidad de resultados en los estudios acerca de la prevalencia del TEPT en supervivientes de un desastre natural. Por ejemplo, tras los terremotos de Taiwán en 1999 como en Grecia el mismo año se encontraron prevalencias de 4,4\% (Wu et al., 2006) y 4,5\% (Roussos et al., 2005) respectivamente en los grupos de supervivientes encuestados. Por otro lado, tras el terremoto de California en 1994 (McMillen, North \& Smith, 2000) y el tsunami asiático del año 2004 (Dewaraja \& Kawamura, 2006), se encontraron prevalencias de un $48 \%$ de sintomatología postraumática y un $42 \%$ de TEPT respectivamente. John, Russell y Russell (2007) revelaron incluso una prevalencia de 70,7\% para el TEPT agudo en niños tamiles del sur de la India afectados por el mismo tsunami. Con estos resultados, si bien se espera un aumento en el diagnóstico de TEPT en la población chilena tras el terremoto de febrero de 2010, no es posible predecir con certeza cuanto será ese incremento. Sí es posible señalar que la prevalencia del TEPT en la población chilena, antes del terremoto, era de $2,5 \%$ en hombres y de $6,2 \%$ en mujeres (Pérez et al., 2009), lo que podría servir de línea base para futuros estudios.

Otros estudios han enfatizado la detección precoz de la población vulnerable a fin de intervenir tempranamente (Dewaraja \& Kawamura, 2006; Karakaya, Agaoglu, Coskun, Sismanlar \& Yildiz Oc, 2004; Ranawaka \& Dewaraja, 2006). Algunos factores de vulnerabilidad que se han encontrado se exponen a continuación. John et al., (2007) encontraron que el TEPT tras el tsunami asiático era más frecuente y grave en mujeres que han estado expuestas a la pérdida de vidas o bienes. Roussos et al., (2005) plantean que en la severidad del TEPT influía la experiencia subjetiva (miedo a morir o ser herido) y objetiva del terremoto (pérdidas humanas, materiales, daño físico). Tural et al., (2001) plantean como factores de vulnerabilidad, el sentir miedo extremo a la muerte durante el terremoto, ser mujer, sufrir lesiones físicas graves y la muerte de parientes de primer grado. Dewaraja y Kawamura (2006) resaltan a la experiencia de la destrucción de una casa y la propiedad sin posibilidad de reparación, ser testigo de la muerte de una persona cercana, estar sin comida y agua, la separación de la familia y haber sido lesionado.

\section{Intervenciones psicológicas}

Uno de los objetivos principales de la intervención psicológica en supervivientes de una catástrofe no es sólo disminuir la sintomatología asociada, sino además mejorar la calidad de vida, generalmente muy alterada a consecuencia de un desastre natural.

Por otro lado, la necesidad de intervención puede darse en cualquiera de las etapas del proceso, inicialmente para dar soporte psicológico frente a la crisis y para prevenir la aparición de síntomas, meses después para reducir esta sintomatología y posibilitar la adaptación a los cambios, a largo plazo para dotar a las personas de herramientas que le permitan sobrellevar su nuevo estilo de vida y afrontar dificultades posteriores.

Sin embargo, los modelos empíricamente validados se orientan más al tratamiento de los síntomas que a la prevención de los mismos y, como ya se ha señalado, la mayoría de los estudios acerca de los efectos psicológicos de un desastre natural, concluyen que es necesaria una intervención temprana para prevenir la ocurrencia de distintos problemas psicológicos en la medida que el tiempo avanza.

Entre las terapias utilizadas que han demostrado su eficacia en el tratamiento del TEPT se encuentran la psicoterapia breve centrada en el trauma/duelo, utilizada en adolescentes supervivientes del terremoto de Armenia en 1988, (Goenjian et al., 1997); técnicas de exposición en simulador de movimientos sísmicos, utilizada por Basoglu, Livanou y Salcioglu (2003) en supervivientes del mismo terremoto; terapia cognitiva centrada en la interpretación de los recuerdos del trauma (Lommen, Sanders, Buck y Arntz, 2009) y desensibilización y reprocesamiento por movimientos oculares o EMDR (Fernández, 2008) en supervivientes del tsunami de Sri-Lanka y Tailandia el año 200, y terapia cognitivo-conductual grupal en niños entre 8 y 12 años supervivientes del terremoto de Atenas en 1999 (Giannopolu et al., 2006).

En cuanto a la prevención del TEPT, los estudios acerca de la intervención temprana en personas sometidas a experiencias traumáticas de cualquier género han entregado conclusiones controvertidas. De partida, Shinfuko (2000) afirma, refiriéndose al terremoto de Kobe, Japón, el año 1995, que lo más apreciado por las víctimas fue el apoyo para su vida diaria en lugar de profesionales de salud mental. Las víctimas compartieron sus experiencias más bien con los voluntarios que les ayudaban. La labor de los profesionales de salud mental fue preparar lineamientos simples para los voluntarios sobre cómo escuchar, alentar y mantener la confidencialidad.

Una de las técnicas preventivas más estudiadas es el "debriefing" que consta de una sola sesión, dirigida por un especialista, y que se ha utilizado ampliamente por más de 25 años para ayudar a las personas a afrontar este tipo de sucesos. El debriefing proporciona al paciente un espacio catártico para que desahogue sus penas y no las reprima (Vera, 2005). Sin embargo, diversos metaanálisis han llegado a concluir que este tipo de intervención no genera diferencias significativas con los grupos de control (van Emmerik, Kamphuis, Hulsbosch \& Emmelkamp, 2002) o 
que incluso genera daño iatrogénico en los participantes (Aulagnier, Verger \& Rouillon, 2004; Sijbrandij, Olff, Reitsma, Carlier \& Gersons, 2006). Aulagnier et al., (2004) proponen, para explicar este resultado, que el debriefing implica una reexposición al recuerdo del trauma, que puede interferir con el curso natural de la recuperación.

Si bien no existe evidencia categórica sobre los efectos preventivos de algunas intervenciones psicológicas sobre el TEPT, existen otras investigaciones que han abordado este tema y que resaltan la importancia de la expresión emocional y el apoyo social en la prevención del TEPT. Por otro lado, la terapia narrativa desarrollada principalmente por White y Epston (1993) ha sido aplicada para la prevención del TEPT en distintas poblaciones, entre ellos, mujeres que han sido diagnosticadas con cáncer de mama (García \& Rincón, 2009). A continuación se revisaran algunos de estos estudios.

\section{Expresión emocional}

Distintas investigaciones guiadas por Pennebaker o realizadas siguiendo sus postulados, han demostrado que la expresión emocional de los significados asociados al trauma previene a largo plazo problemas en la salud y genera un aumento en el funcionamiento del sistema inmunológico, entre otras consecuencias (Owen et al., 2006; Pennebaker, 1997; Pennebaker, Kiecolt-Glaser \& Glaser, 1988; Pennebaker \& Seagal, 1999; Petrie, Booth $\&$ Pennebaker, 1998). Pennebaker (2004) ha desarrollado un ejercicio de escritura emocional que ha sido aplicado en distintos contextos y que, de acuerdo a la revisión de Cabrera (2006), ha provocado mejoras en la salud física y psicológica de los participantes.

Por otra parte, Cabrera (2006) señala que algunas formas de escribir parecen mostrar mejores efectos en la salud que otras. Por ejemplo, es importante identificar y etiquetar adecuadamente las emociones negativas y positivas, construir una historia coherente y significativa de los hechos traumáticos y poder narrar la experiencia desde diversas perspectivas. Cabrera (2006), advierte que las personas que más se beneficiarían de los ejercicios de escritura son aquellos que han vivido una situación traumática y tienen dificultades para confrontar los hechos con otras personas y por ello mantienen en secreto sus dificultades.

\section{Apoyo social}

El apoyo social se puede concebir como el sentimiento de ser apreciado y valorado por otras personas y de pertenecer a una red social (Barra, 2004), y parece influir positivamente en una mejor calidad de vida, baja morbilidad psicológica y mayor sobrevida. Su importancia ha sido demostrada por diversos estudios que indican, por ejemplo, que la falta de apoyo social es un factor de riesgo para la aparición del TEPT (Brewin, Andrews \& Valentine,
2000) o que resulta un buen predictor para la aparición de este trastorno en supervivientes de un terremoto (Altindag, Ozen \& Sir, 2005).

Armenian et al., (2000) encontraron que, junto a un mayor nivel educacional y la posibilidad de hacer amigos tras la tragedia, el apoyo inmediato fue un importante factor protector para prevenir el TEPT en supervivientes del terremoto de Armenia el año 1988, medido el TEPT dos años después del desastre. Concluyen que el apoyo temprano a los sobrevivientes con altos niveles de pérdidas puede prevenir el TEPT.

\section{Terapia narrativa}

Ante la relevancia de la expresión emocional y del apoyo social, el modelo de intervención narrativo, cuyo desarrollo se ha visto también influido por las investigaciones de Pennebaker (Galarce, 2003; Tarragona, 2003), podría ser un modelo útil para prevenir síntomas del TEPT en supervivientes de catástrofes naturales. Sin embargo, la relativa juventud de este enfoque ha proporcionado menos evidencias acerca de sus resultados. Dentro de éstas, destacan la revisión de O'Kearney y Perrott (2006), quienes analizan 19 estudios que describían las narrativas del trauma en personas con diagnóstico o sintomatología TEPT. Kaminer (2006), a su vez, hace una revisión de otros estudios que se centran en la influencia de la narración del trauma en la recuperación del TEPT, enfocándose en determinar cuáles son los procesos psicológicos implicados en cada uno de ellos, planteando que el proceso implicado específicamente en la terapia narrativa es la identificación del propósito y valor de la adversidad.

La importancia de la asignación de significado a las experiencias traumáticas, puede hallarse en un estudio de Norman (2000) en el que se concluye que si bien la exposición a sucesos traumáticos puede generar TEPT, no todas las personas desarrollan el síndrome. La diferencia, según Norman (2000), sería "que algunas personas son hábiles en encontrar significado para sus experiencias horrorosas" (p. 305). El intento de dar un sentido a experiencias negativas ha sido resaltado por la reciente psicología positiva, escuela que incorpora constructos como el de resiliencia, cualidad que presentan algunas personas para sobreponerse a la adversidad y a los acontecimientos traumáticos. El modelo narrativo de White y Epston, coherente con esto, afirma que las experiencias adversas son también historias de resiliencia y supervivencia y que estos aspectos pueden ser ampliados y enriquecidos a través del proceso terapéutico (Kaminer, 2006).

El enfoque narrativo de White y Epston (Epston, 1994; White, 2002a, 2002b; White \& Epston, 1993) se basa en el supuesto de que las narrativas no representan la identidad y los problemas de la personas; más bien las narrativas son la identidad y los problemas. Al respecto, Carr (1998) señala que los problemas humanos aparecen y se mantienen gracias 
PREVENCIÓN DE TRASTORNO DE ESTRÉS POSTRAUMÁtico EN SUPERVIVIENTES DEL TERREMOTO DE ChILE DE FEBRERO DE 2010 :

UNA PROPUESTA DE INTERVENCIÓN NARRATIVA

Tabla 1. Resumen de la propuesta de intervención

\begin{tabular}{|c|c|}
\hline SESIÓN/ETAPA & ACTIVIDADES \\
\hline 1 & $\begin{array}{l}\text { - Encuadre terapéutico } \\
\text { - Exploración del problema } \\
\text { - Formulación de objetivos terapéuticos } \\
\text { Tarea: } \\
\text { - Ejercicio de escritura emocional }\end{array}$ \\
\hline 2 & $\begin{array}{l}\text { - Análisis y normalización de las reacciones psicológicas post-terremoto } \\
\text { - Revisión de la tarea de escritura emocional } \\
\text { Tarea: } \\
\text { - Ejercicio de Conexión con red de apoyo social }\end{array}$ \\
\hline 3 & $\begin{array}{l}\text { - Revisión de la tarea de conexión con red de apoyo social } \\
\text { - Discusión sobre las redes de apoyo. } \\
\text { - Exploración de narrativas alternativas } \\
\text { Tarea: } \\
\text { - Buscar acontecimientos de la vida personal en los cuales hayan vencido un obstáculo }\end{array}$ \\
\hline 4 & $\begin{array}{l}\text { - Revisión de la tarea de búsqueda de acontecimientos } \\
\text { - Discusión sobre las historias de supervivencia y superación en la vida de los consultantes } \\
\text { - Participación en la construcción de una nueva narrativa que incorpora los elementos que han surgido a lo } \\
\text { largo de las sesiones. }\end{array}$ \\
\hline
\end{tabular}

a las historias opresivas que dominan la vida de las personas. Pero estos relatos no sólo determinan el significado atribuido a sus vivencias, sino que también determinan qué aspectos de la experiencia vivida seleccionan para asignarles un significado.

El objetivo de la terapia narrativa es ayudar al cliente a reescribir su vida, incorporando a su relato pedazos de la historia que han sido marginados de la experiencia, acontecimientos que constituyen excepciones a la narrativa actual; entonces la persona será capaz de dar un nuevo significado a su vida pasada y proyectar un futuro menos opresivo que el que manifiesta en la actualidad. Además, este enfoque señala que los nuevos significados cobran mayor valor en cuanto son transmitidos y compartidos con la red social (familia, amigos) que rodea y sostiene al paciente, propiciando, por lo tanto, diversas instancias en donde es posible establecer esta conexión, ya sea en forma simbólica (cartas terapéuticas) o en forma directa (foros, talleres, encuentros familiares).

El enfoque narrativo se basa en el principio de que las personas categorizan sus experiencias a través del lenguaje. Sin embargo, al momento de narrar la experiencia, ciertas partes quedan fuera porque son menos atendidas que otras. Sin embargo, cuando logran recordar éstas partes, las personas son capaces de formular una historia más completa de su experiencia. Si se anima a los pacientes a atender a las partes marginadas de su experiencia, pueden crear historias íntegras y atribuir nuevos significados. En otras palabras, la terapia narrativa invita a los supervivientes a involucrarse en una revaloración, construir nuevos significados e integrarlos en su experiencia.
Con estos antecedentes, parece ser que el enfoque narrativo podría convertirse en un modelo adecuado para prevenir o reducir los síntomas de TEPT, haciéndose necesario realizar estudios para probar su eficacia. Uno de esos estudios fue desarrollado por García y Rincón (2009) para prevenir la aparición de TEPT en pacientes recién diagnosticadas con cáncer de mama. Para ello, se diseñó un taller de cuatro sesiones, con grupo control, obteniendo diferencias significativas entre ambos grupos en sintomatología postraumática, tres meses después del diagnóstico, siendo menor en el grupo que participó en el taller.

\section{Propuesta de intervención narrativa}

En base al estudio de García y Rincón (2009), los textos teóricos y prácticos acerca de terapia narrativa, el protocolo individual de Pérez y Vásquez (2001) para la intervención narrativa de pacientes con TEPT, la propuesta de trabajo grupal de Petersen et al., (2005) para la prevención del TEPT en pacientes con cáncer y la propuesta de ejercicio de escritura emocional de Pennebaker (Cabrera, 2006; Fernández, Páez y Pennebaker, 2004; Pennebaker, 2004), hemos diseñado una propuesta de intervención narrativa para supervivientes del terremoto y tsunami que afectó a Chile el 27 de febrero de 2010. Un resumen de este procedimiento, que podría adaptarse tanto para la atención individual como grupal, se revisa en la tabla 1.

Por otro lado, es necesario indicar que el terapeuta que adopta una posición narrativa se caracteriza por su intento de empoderar al cliente como experto en su vida y en el problema que lleva a terapia, la externalización del problema, la deconstrucción de su relato intentando reconocer narrativas alternativas excluidas de su relato principal, la búsqueda de 
excepciones o recursos, una reconstrucción o reescritura de su narrativa incorporando los nuevos relatos que han aparecido a lo largo de la terapia y la transmisión de su nuevo relato a personas significativas de su red social..

Es preciso señalar además que las sugerencias de intervención que se señalan a continuación representan una guía que puede ser útil para el terapeuta, pero no corresponde a un protocolo de intervención, por cuanto la postura narrativa implica la capacidad del terapeuta de adaptarse al lenguaje, al estilo y a la narrativa del cliente, y construir este nuevo relato a partir de la conversación que se suscite en la terapia, por lo que indicaciones rígidas y protocolizadas, no serían coherentes con el trabajo terapéutico que proponemos.

\section{Sesión/etapa 1}

El terapeuta debe acoger al cliente con una actitud de facilitación de la expresión cognitiva y emocional de la experiencia del terremoto, sin forzar el diálogo. No se recomienda la aplicación de pruebas psicológicas ni una indagación anamnésica acabada por cuanto el objetivo del primer encuentro es la generación de una alianza terapéutica, la exploración del problema y la co-construcción de objetivos terapéuticos. La excepción sería dada si la intervención estuviera inserta en un proceso de investigación, para lo cual se recomienda que la búsqueda de antecedentes laterales y toma de pruebas se realice antes de la primera sesión

La exploración del problema debe implicar la externalización de la experiencia del terremoto o de las reacciones psicológicas como "cosas que le han sucedido" y no como características personales. Por ejemplo, sugerimos hablar más bien de ese "estado depresivo que ha afectado a su vida desde el día del terremoto" y no de su "carácter depresivo".

También debe procurarse la deconstrucción del relato de la experiencia, generando preguntas reflexivas que no impliquen un cuestionamiento o debate, sino más bien desde una posición de auténtica curiosidad e ingenuidad por conocer detalles de su experiencia. Preguntas posibles de formular serían: ¿Cómo te ha afectado el terremoto? ¿Cuáles son las áreas de tu vida que se han visto más alteradas? ¿Cuáles son las áreas de tu vida que se han visto menos alteradas? ¿Por qué crees tú que el terremoto no ha afectado tanto esas áreas? ¿Qué has hecho tú para mantener los efectos del terremoto al margen en esas áreas? ¿Quiénes te han ayudado a mantener a raya los efectos del terremoto en esas áreas?

Al finalizar la sesión se propone como tarea un ejercicio de escritura emocional, las instrucciones se anexan en la tabla 2.

\section{Sesión/etapa 2}

Se sostiene una conversación destinada a explorar los efectos psicológicos del terremoto. Se sugiere intentar normalizar algunas de esas respuestas recalcando que corresponden a reacciones normales frente a un acontecimiento anormal y no a respuestas patológicas aunque lo hagan sufrir y entorpezcan su vida. Recalcar también a fin de evitar que el consultante sienta que se minimiza su problema, que si bien su respuesta es normal, su intensidad, duración o pertinencia es una dificultad que necesita ser superada para mejorar la calidad de vida. Puede ayudarse a través de alguna metáfora, para lo cual sugerimos la siguiente: un automóvil sin alarma, corre el riesgo de robo; una alarma calibrada avisa los golpes a través de un sonido estridente, pero a veces se desajustan y entonces se activan al menor movimiento, sin que exista un verdadero riesgo, agotando la batería o impidiendo el sueño de su dueño o sus vecinos. No podemos estar sin alarma, pero tampoco podemos permitir que se active a cada instante y por cualquier cosa.

Un cuadro de normalizaciones posibles se adjunta en la tabla 3, tomado de Pérez y Vásquez (2001).

El análisis de la tarea de escritura emocional debería orientarse a evaluar la experiencia de escribir, por ejemplo, preguntando en qué medida ha expresado sus sentimientos y pensamientos más íntimos, si ha sentido tristeza, malestar o felicidad y si el ejercicio ha sido valioso y significativo. En la conversación se busca deconstruir el relato de la experiencia y rastrear significados.

Al finalizar la sesión, se propone una segunda tarea destinada a reconocer sus fuentes de apoyo social, a través de una instrucción como la siguiente: "Pregúntele a alguien cercano, ya sea familiar o amigo, sobre lo que piensa y siente hacia su reacción frente a las consecuencias del terremoto. Sólo debe escuchar, sin cuestionar o corregir lo que dice. Si lo desea, puede hacer este ejercicio con más de una persona significativa".

\section{Sesión/etapa 3}

Se inicia revisando la tarea, consultando cómo se sintió el consultante al escuchar a esta persona, rescatar elementos de ese relato que le parezcan importantes o que no haya considerado relevantes en su propio relato o se le hayan pasado por alto. Explorar a continuación la relevancia que han tenido otras personas para apoyarla, ya sea a través del auxilio para la satisfacción de necesidades básicas, para mantenerla informada acerca de sus inquietudes, para brindarle apoyo emocional cuando lo ha requerido o para darle soporte espiritual o religioso.

La exploración de narrativas alternativas se realiza a partir de preguntas que busquen conocer otras visiones $u$ opiniones de seres cercanos o sus fuentes de apoyo sobre los mismos hechos, y deconstruir la naturaleza de esos relatos, con preguntas como las sugeridas a continuación: ¿En qué 


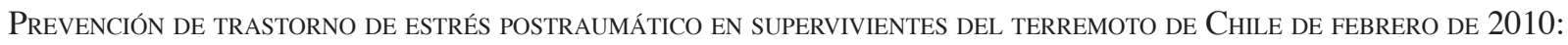

UNA PROPUESTA DE INTERVENCIÓN NARRATIVA

Tabla 2. Instrucciones para el ejercicio de escritura emocional.

DÍA 1

- Reflexione unos minutos acerca de lo que usted personalmente ha sentido, pensado y hecho en relación al día del terremoto y/o tsunami.

- Luego de haber reflexionado, escriba lo que ha pensado en un mínimo de una plana y un máximo de tres planas

- $\quad$ No se preocupe de la redacción o la ortografía.

- $\quad$ Piense que lo escrito es absolutamente personal o que es un testimonio anónimo que está transmitiendo a una persona que no ha vivido esa experiencia.

- En su escrito, manifieste cómo le ha afectado el terremoto y/o tsunami realmente. Por ejemplo, podría ser beneficioso simplemente escribir sobre el terremoto y/o tsunami en sí mismo, cómo se sintió en el momento en que vivió esta experiencia y cómo le hace sentir ahora.

DÍA 2

- Siguiendo las mismas instrucciones generales del Día 1, en este día profundice por escrito en sus sentimientos y pensamientos más íntimos revisando el efecto que el terremoto y/o tsunami ha tenido en las distintas áreas de su vida.

DÍA 3

- Siguiendo las mismas instrucciones generales del día 1, en este tercer día intente analizar desde diferentes puntos de vista los acontecimientos relacionados con el terremoto y/o tsunami. Es decir, intente verlo desde el lado negativo, positivo o como usted DÍA 4 cree que lo ven las personas más cercanas. Y póngalo por escrito.

- Este último día reflexione sobre lo escrito en los días anteriores valorando si hay asuntos que aún no han sido enfrentados, y si ha aprendido, perdido y ganado algo como resultado del terremoto y/o tsunami. Además explore en qué medida esta experiencia marcará su comportamiento futuro. Y escríbalo.

Tabla 3. Ejemplos de normalización para los síntomas del TEPT

\begin{tabular}{ll}
\hline SINTOMAS & NORMALIZACIÓN PROPUESTA \\
\hline $\begin{array}{l}\text { Síntomas intrusivos (flashbacks, pesadillas, } \\
\text { rumiación) }\end{array}$ & $\begin{array}{l}\text { Intentos recurrentes de dar sentido a la experiencia, intentos espontáneos de la mente } \\
\text { para entender y asimilar lo ocurrido, de buscar un final. }\end{array}$ \\
$\begin{array}{l}\text { Síntomas de anestesia emocional, de despersonali- } \\
\text { zación o de extrañeza }\end{array}$ & $\begin{array}{l}\text { Necesidad de la mente de desconectarse temporalmente de la realidad, de poner algo } \\
\text { de distancia respecto al mundo y darse un tiempo de respiro y recuperación }\end{array}$ \\
$\begin{array}{l}\text { Angustia ante situaciones parecidas y el deseo de } \\
\text { evitarlas }\end{array}$ & $\begin{array}{l}\text { Un modo natural de protegerse. Un modo de permitirse ir afrontando lentamente } \\
\text { las cosas. Dosificación necesaria del dolor ante la pérdida }\end{array}$ \\
Ganas de estar solo y aislarse & $\begin{array}{l}\text { Intentos por no perder el control y mantener la calma interior } \\
\text { Hiperactivación y alarma }\end{array}$ \\
$\begin{array}{l}\text { Actitud defensiva, útil para sobrevivir. Agotadora, cuando se lleva al extremo, pero } \\
\text { cuyo fin es proteger. Una vez pasada la amenaza ya no sería necesario mantenerla, } \\
\text { aunque a veces tome un tiempo desactivarla progresivamente }\end{array}$ \\
\hline
\end{tabular}

medida crees que la visión de $\mathrm{x}$ acerca de tu experiencia del terremoto complementa tu propia versión? ¿En qué antecedentes crees que $\mathrm{x}$ se basa para sostener su opinión? ¿Y cuál es tu opinión acerca de esos antecedentes? ¿Si le preguntáramos a x sobre tus fortalezas y debilidades tras el terremoto, qué crees tú que nos diría él/ella? ¿Y qué piensas tú acerca de estas fortalezas y debilidades?

La sesión concluye con una tarea destinada a que el consultante recupere recursos personales y sociales que le hayan permitido superar otras dificultades en sus vidas. Como instrucción se sugiere lo siguiente: "Recuerde de aquí a la próxima sesión algunos eventos en su vida en el cual usted enfrentó un problema o dificultad y logró vencerla o salir adelante. Es importante que intente recordar que hizo, pensó o sintió, y a quien recurrió, para superar cada uno de estos problemas o dificultades".

\section{Sesión/etapa 4:}

Al iniciar la sesión, se le invita a compartir las experiencias solicitadas en la tarea. Se le invita a expresar cómo se sintió durante su rememoración y se le insta a reconocer "de qué se percataron" realizando esta tarea. 
A continuación, se conversa sobre las diversas experiencias en la historia de su vida, en las que hayan demostrado su capacidad para enfrentarse o reponerse frente a la adversidad, centrando el diálogo en descubrir y ampliar aquellas cualidades que les permitieron salir adelante o en los aprendizajes que obtuvieron tras vivir estas experiencias. Se utiliza el mismo lenguaje deconstructivo para profundizar en las historias y proyectar sus aprendizajes hacia el futuro inmediato, promoviendo conversaciones que busquen internalizar estos recursos y empoderar al consultante.

A partir del recuento de los relatos y experiencias complementarias a su narrativa original, que surgieron a lo largo de la terapia, se sostiene una conversación destinada a construir una nueva narrativa que incorpore estos elementos, buscando conectar este nuevo relato con los desafíos que le esperan en el futuro.

En sesiones posteriores, si las hubiera, se sugiere utilizar estos recursos del cliente para que éste afronte otros problemas que le estén afectando.

\section{Discusión}

Existen suficientes investigaciones que exploran la prevalencia y factores de riesgo del TEPT tras desastres naturales. Tal información es útil para identificar a los grupos vulnerables hacia quienes debe dirigirse la asistencia psicológica.

La mayoría de las intervenciones psicológicas revisadas se han centrado principalmente en reducir la sintomatología postraumática cuando ya está desarrollada la patología. Nosotros creemos contar con información suficiente para diseñar y ejecutar intervenciones preventivas, sobre todo cuando han trascurrido menos de dos meses desde el terremoto, a fin de que dichas manifestaciones no aparezcan. Al estudiar las intervenciones preventivas que se han utilizado, descubrimos resultados contradictorios en cuanto a su efectividad, con evidencia de daño iatrogénico como en el debriefing, aun hoy muy popular para el apoyo inicial de los supervivientes. Por otro lado, la imperiosa necesidad de obtener ayuda material en los primeros tiempos tras un terremoto, dificulta la acción directa de los profesionales de salud mental, haciéndose más recomendable capacitar, para que sean una oportunidad de apoyo emocional, a los voluntarios que entregan ayuda directa, tal como se sugiere en los Primeros Auxilios Psicológicos (Ruzek et al., 2007).

Una vez satisfechas las personas en sus necesidades más básicas, aumenta el interés por recibir apoyo psicológico profesional. Hemos visto en estos días, quienes vivimos en la zona de catástrofe y nos dedicamos a la psicología clínica, un aumento significativo de la demanda de atención para problemas psicológicos relacionados con los efectos del terremoto y tsunami. Frente a dicha demanda, y considerando el poco tiempo transcurrido desde el sismo, proponemos un modelo de intervención breve, basado en la terapia narrativa de White y Epston (1993) y enriquecido con los hallazgos acerca de la importancia de la expresión emocional para la reducción de morbilidad psicológica y física en pacientes sometidos a situaciones traumáticas, así como los estudios que resaltan la relevancia de establecer redes de apoyo social desde los primeros días para los supervivientes de catástrofes. Ambas, expresión emocional y apoyo social, coherentes con las propuestas y principios de la terapia narrativa.

Proponemos entonces un modelo de intervención que puede servir de guía para quienes estamos involucrados en esta tarea, un modelo susceptible de corrección y ampliación, pero que puede significar un aporte para ayudar a los supervivientes de este desastre. Este modelo ha sido utilizado previamente por el autor principal de este artículo para la prevención del TEPT en mujeres recién diagnosticadas con cáncer de mama, demostrando su efectividad. Si bien la propuesta que se presenta en este artículo no tiene la pretensión de convertirse en un protocolo estandarizado, sus lineamientos principales son susceptibles de ser sometidos a prueba empírica.

\section{Referencias}

Altinda, A., Ozen, S., \& Sir, A. (2005). One-year follow-up study of posttraumatic stress disorder among earthquake survivors in Turkey. Comprehensive Psychiatry, 46, 328-333.

Armenian, H., Morikawa, M., Melkonian, A., Hovanesian, A., Haroutunian, N., Saigh, P., Akiskal, K., \& Akiskal, H. (2000). Loss as a determinant of PTSD in a cohort of adult survivors of the 1988 earthquake in Armenia: Implications for policy. Acta Psychiatrica Scandinavica, 102, 58-64.

Aulagnier, M., Verger, P., \& Rouillon, F. (2004). Efficiency of psychological debriefing in preventing post-traumatic stress disorders. Revue d'Épidémiologie et de Santé Publique, 52, 67-79.

Barra, E. (2004). Apoyo social, estrés y salud. Psicología y Salud, 14, 237-243.

Brewin, C., Andrews, B., \& Valentine, J. (2000). Meta-analysis of risk factors for posttraumatic stress disorder in trauma-exposed adults. Journal of Consulting and Clinical Psychology, 68, 748-766.

Basoglu, M., Livanou, M., \& Salcioglu, E. (2003). A single session with an earthquake simulator for traumatic stress in earthquake survivors. American Journal of Psychiatry, 160, 788-790.

Cabrera, E. (2006). Efectos terapéuticos de la escritura emocional o expresiva.Hojas Informativasdel@s Psicólog@s de Las Palmas, (89), 9-12. Extraído el 28 de octubre de 2007 desde: www.coplaspalmas. org/07biblio/psicot/psicot31.pdf

Carr, A. (1998). Michael White's narrative therapy. Contemporary Family Therapy, 20, 485-503.

Dewaraja, R., \& Kawamura, N. (2006). Trauma intensity and posttraumatic stress: Implications of the tsunami experience in Sri Lanka for the management of future disasters. International Congress Series, 1287, 69-73.

Epston, D. (1994). Obras escogidas. Barcelona: Gedisa.

Fernández, I. (2008). EMDR after a critical incident: Treatment of a tsunami survivor with acute posttraumatic stress disorder. Journal of EMDR Practice and Research, 2, 156-159.

Fernández, I., Páez, D., y Pennebaker, J. (2004). Escritura expresiva, deber de memoria y afrontamiento tras el impacto del 11-M: Un estudio experimental. Ansiedad y Estrés, 10, 233-245.

Gaborit, M. (2001). Desastres y trauma psicológico. Pensamiento psicológico, 2, 15-39. 
Galarce, E. (2003). Psicología Narrativa: Una revisión de sus aspectos teóricos y sus alcances terapéuticos. Tesina presentada para optar al Grado de Licenciado en Psicología, Facultad de Ciencias Sociales, Universidad de Belgrano, Buenos Aires, Argentina.

García, F. \& Rincón, P. (2009). Prevención de Sintomatología Postraumática en Mujeres Diagnosticadas con Cáncer de Mama: Evaluación Preliminar de un Modelo de Intervención Narrativo. Tesis para optar al grado de Magíster en Psicología de la Salud, Escuela de Psicología, Universidad de Concepción, Chile.

Giannopoulou, I., Dikaiakou, A., \& Yule, W. (2006). Cognitive-behavioural group intervention for PTSD symptoms in children following the Athens 1999 earthquake: A pilot study. Clinical Child Psychology and Psychiatry, 11, 543-553.

Goenjian, A., Karayan, I., Pynoos, R., Minassian, D., Najarian, L., Steinberg, A., \& Fairbanks, L. (1997). Outcome of psychotherapy among early adolescents after trauma. American Journal of Psychiatry, 154, 536-542.

John, P., Russell, S., \& Russell, P. (2007). The prevalence of posttraumatic stress disorder among children and adolescents affected by tsunami disaster in Tamil Nadu. Disaster Management \& Response, 5, 3-7.

Kaminer, D. (2006). Healing processes in trauma narratives: A review. South Africans Journal of Psychology, 36, 481-499.

Karakaya, I., Agaoglu, B., Coskun, A., Sismanlar, S. \& Yildiz Oc, O. (2004). The symptoms of PTSD, depression and anxiety in adolescent students three and a half years after the Marmara earthquake. Turk Psikiyatri Dergisi. 15, 257-263.

Lommen, M., Sanders, A., Buck, N., \& Arntz, A. (2009). Psychosocial predictors of chronic Post-Traumatic Stress Disorder in Sri Lankan tsunami survivors. Behaviour Research and Therapy, 47, 60-65.

Marcos, E., Medina, J., Pérez-Iñigo, J., Robles, J., \& Gómez-Trigo, J. (2002). Conductas Individuales y Reacciones Ante Situaciones de Catastrofe. III Congreso Virtual de Psiquiatría, Interpsiquis, Universitat de Barcelona, España. Extraído en enero 26, 2009 disponible en www. psiquiatria.com/articulo/ansiedad/estres/4799.

McMillen, J. C., North, C., \& Smith, E. (2000). What parts of PTSD are normal: intrusion, avoidance, or arousal? Data from the northridge, california, earthquake. Journal of Traumatic Stress, 13, 57-79.

Norman, J. (2000). Constructive narrative in arresting the impact of posttraumatic stress disorder. Clinical Social Work Journal, 28, 303-319.

O'Kearney, R., \& Perrott, K. (2006). Trauma narratives in posttraumatic stress disorder: A review. Journal of Traumatic Stress, 19, 81-93.

Owen, J., Giese-Davis, J., Cordova, M., Kronenwetter, C., Golant, M., \& Spiegel, D. (2006). Self-report and linguistic indicators of emotional expression in narratives as predictors of adjustment to cancer. Journal of Behavioral Medicine, 29, 335-345.

Páez, D., Arroyo, E., \& Fernández, I. (1995). Catástrofes, situaciones de riesgo y factores psicosociales. Mapfre y Seguridad, 57, 43-45.

Pennebaker, J. (1997). Writing about emotional experiencies as a therapeutic process. Psychological Science, 8, 162-166.

Pennebaker, J. (2004). Writing to heal: a guided journal for recovering from trauma and emotional upheaval. Oakland, CA: New Harbinger Press.

Pennebaker, J., Kiecolt-Glaser, J., \& Glaser, R. (1988). Disclosure of traumas and immune function: Health implications for psychotherapy. Journal of Consulting and Clinical Psychology, 56, 239-245.

Pennebaker, J., \& Seagal, J. (1999). Forming a story: The health benefits of narrative. Journal of Clinical Psychology, 55, 1243-1254.
Pérez, P., \& Vásquez, C. (2001). Reconceptualizar la psicología del trauma desde los recursos positivos: Una visión alternativa. 2 do. Congreso Virtual de Psiquiatría, Interpsiquis, Universitat de Barcelona, España.

Pérez, C., Vicente, B., Zlotnick, C., Kohn, R., Johnson, J., Saldivia, S., \& Rioseco, P. (2009). Estudio epidemiológico de sucesos traumáticos, trastorno de estrés post-traumático y otros trastornos psiquiátricos en una muestra representativa de Chile. Salud Mental, 32, 145-153.

Petersen, S., Bull, C., Propst, O., Dettinger, S., \& Detwiler, L. (2005). Narrative therapy to prevent illness-related stress disorder. Journal of Counseling and Development, 83, 41-47.

Petrie, K., Booth, R., \& Pennebaker, J. (1998). The inmunological effects of thought suppression. Journal of Personality and Social Psychology, $75,1264-1272$.

Ranawaka D. \& Dewaraja, R. (2006). Tsunami counselling project of the Sri Lanka National Institute of professional counsellors. International Congress Series, 1287, 79-81.

Roussos, A., Goenjian, A., Steinberg, A., Sotiropoulou, C., Kakaki, M., Kabakos, C., Karagianni, S., \& Manouras, V. (2005). Posttraumatic stress and depressive reactions among children and adolescents after the 1999 earthquake in Ano Liosia, Greece. American Journal of Psychiatry, 162, 530-537.

Ruzek, J., Brymer, M., Jacobs, A., Layne, C., Vernberg, E., \& Watson, P. (2007). Psychological first aid. Journal of Mental Health Counseling, 29, 17-49.

Sassón, M. (2004). Catástrofes y Salud Mental. Abordajes Teóricos y Modalidades de Intervención. Tesina para optar al Grado de Licenciado en Psicología, Facultad de Humanidades, Universidad de Belgrano, Argentinas.

Shinfuku, N. (2002). Disaster mental health: Lessons learned from the Hanshin Awaji earthquake. World Psychiatry, 1, 158-159.

Sijbrandij, M., Olff, M., Reitsma, J., Carlier, I., \& Gersons, B. (2006). Emotional or educational debriefing after psychological trauma. The British Journal Of Psychiatry, 189, 150-155.

Tarragona, M. (2003). Escribir para re-escribir historias y relaciones. Psicoterapia y Familia, 16 (1), 45-54.

Tural, U., Aybar Tolun, H., Karakaya, I., Erol, Y., Yildiz, M., \& Erdoğan, S. (2001). Predictors of current comorbid psychiatric disorders with posttraumatic stress disorder in earthquake survivors. Turkish Journal of Psychiatry, 12, 175-183.

Van Emmerik, A., Kamphuis, J., Hulsbosch, A., \& Emmelkamp, P. (2002). Single session debriefing after psychological trauma: A meta-analysis. Lancet, 360, 766-771.

Vera, B. (2005). Trauma y ventilación emocional. ¿Mito o realidad? Cuadernos de Crisis, 2, 7-13.

White, M. (2002a). El enfoque narrativo en la experiencia de los terapeutas. Barcelona: Gedisa.

White, M. (2002b). Reescribir la vida. Barcelona: Gedisa.

White, M., \& Epston, D. (1993). Medios narrativos para fines terapéuticos. Barcelona: Gedisa.

Wu, H., Chou, P., Chou, F., Su, C., Tsai, K., Ou-Yang, W., Su, T., Chao, S., Sun, W., \& Chen, M. (2006). Survey of quality of life and related risk factors for a Taiwanese village population 3 years post-earthquake. Australian and New Zealand Journal of Psychiatry, 40, 355-361. 